\title{
Efeitos anestésicos da administração intranasal ou intramuscular de cetamina S+ e midazolam em pomba-rola (Streptotelia sp.) ${ }^{1}$
}

\author{
Suzane L. Beier²*, Ademir C. Rosa², Nilson Oleskovicz², Cláudio R.S. Mattoso² \\ e Aury N. Moraes ${ }^{2}$
}

\begin{abstract}
Beier S.L., Rosa A.C., Oleskovicz N., Mattoso C.R.S. \& Moraes A.N. 2013. [Anesthetic effects of intranasal or intramuscular administration of S+ Ketamine and Midazolam in ring necked dove (Streptotelia sp.).] Efeitos anestésicos da administração intranasal ou intramuscular de cetamina S+ e midazolam em pomba-rola (Streptotelia sp.). Pesquisa Veterinária Brasileira 33(4):517-522. Departamento de Medicina Veterinária Centro de Ciências Agroveterinárias, Universidade do Estado de Santa Catarina, Av. Luiz de Camões 2090, Lages, SC 88520-000, Brazil. E-mail: suzanelb@yahoo.com.br

The intranasal route is a good alternative because is painless and easy to perform in birds. The objective of this study was to evaluate the anesthetic effects of S+ ketamine and midazolam administered by intranasal or intramuscular route in pigeons. Twelve animals were used in a randomized and crossover design. Animals received two treatments with 2-weeks interval. IM group: animals received $20 \mathrm{mg} / \mathrm{kg}$ of S+ ketamine and $3.5 \mathrm{mg} / \mathrm{kg}$ of midazolam by intramuscular route (pectoral muscles); IN group: animals received the same protocol by intranasal route. Parameters evaluated were: onset of action, time of duration in dorsal recumbency; total time of anesthesia and side effects. Statistical analysis was performed using Wilcoxon test and the differences were considered significant when $\mathrm{P}<0.05$. Onset of action was 30 [30-47.5] and 40 [30-50] seconds for IM and IN respectively. Time of duration in dorsal recumbency was 59 [53.25-65] and 63 [37-71.25] minutes for IM and IN respectively, without significant differences between treatments. Total time of anesthesia was 88 [86.2594.5] and 68 [53.5-93] minutes for IM and IN, respectively, with significant difference between groups. The recovery time was lower for IN (15[4.25-19.5] minutes) compared with IM (32 [28.25-38.25] minutes). Two animals of each group presented regurgitation in the recovery period. It was concluded that $S+$ ketamine and midazolam administered intranasal is an acceptable method of drug delivery and can be used to promote anesthesia in pigeons.

INDEX TERMS: Administration route, birds, chemical restraint, S+ Ketamine, midazolam, ring dove, Streptotelia sp.
\end{abstract}

RESUMO.- A via intranasal é uma boa alternativa por ser indolor e de fácil aplicação em aves. 0 objetivo deste estudo foi avaliar os efeitos anestésicos da associação de cetamina S+ e midazolam pela via intranasal (IN) em comparação com a via intramuscular (IM) em pombos. Foram utilizados 12 pombos alocados em dois grupos com 15 dias de intervalo, os quais receberam: grupo IM: $20 \mathrm{mg} / \mathrm{kg}$ de cetamina $\mathrm{S}+$ associada a $3,5 \mathrm{mg} / \mathrm{kg}$ de midazolam pela via intramuscular (musculatura do peito); e grupo IN, mesmo protocolo,

\footnotetext{
${ }^{1}$ Recebido em 22 de agosto de 2012.

Aceito para publicação em 4 de fevereiro de 2013.

${ }^{2}$ Departamento de Medicina Veterinária, Centro de Ciências Agroveterinárias, Universidade do Estado de Santa Catarina (UDESC), Av. Luiz de Camões 2090, Lages, SC 88520-000, Brasil. *Autor para correspondência: suzanelb@yahoo.com.br
}

porém, pela via intranasal. Os parâmetros avaliados foram: período de latência, tempo de duração em decúbito dorsal, tempo total de anestesia, tempo de recuperação e efeitos adversos. Para a análise estatística, empregou-se o teste de Wilcoxon, com as diferenças consideradas significativas quando $\mathrm{P}<0,05$. 0 período de latência obtido foi de 30 [30$47,5]$ e 40 [30-50] segundos para IM e IN, respectivamente. 0 tempo de duração de decúbito dorsal foi de 59 [53,2565] e 63 [37-71,25] minutos para IM e IN, respectivamente, sem diferenças significativas entre os grupos. Com relação à duração total de anestesia, foi observada diferença significativa, com 88 [86,25-94,5] e 68 [53,5-93] minutos para os grupos IM e IN, respectivamente. 0 tempo de recuperação foi mais curto no grupo IN $(15[4,25-19,5])$ comparado ao IM $(32$ [28,25-38,25] minutos). Dois animais de cada 
grupo apresentaram regurgitação na fase de recuperação. Conclui-se que a administração de cetamina S+ e midazolam pela via intranasal é um método aceitável de administração de fármacos e produz anestesia rápida e eficaz em pombos.

TERMOS DE INDEXAÇÃO: Vias de administração, aves, contenção química, cetamina S+, midazolam, pomba-rola, Streptotelia sp.

\section{INTRODUÇÃO}

A anestesia é um desafiador e importante aspecto na medicina e cirurgia de aves (Gunkel \& Lafortune 2005), sendo que a contenção química nestes animais tem por objetivo evitar situações estressantes e de ansiedade (Harrison 1986, Coles 1997) e é necessária para muitos procedimentos a serem realizados, como sexagem, radiografias e colheitas de amostras sanguíneas, entre outros (Vesal \& Zare 2006). Fármacos anestésicos podem ser utilizados na forma de gases (anestesia inalatória) ou por via injetável, sendo que a anestesia inalatória é a preferida em aves por sua segurança, porém, necessita de aparelhos que nem sempre estão disponíveis, os quais também são de alto custo (Moghadam et al. 2009).

Uma grande variedade de fármacos anestésicos e suas combinações são utilizadas para sedação e indução de pássaros (Gunkel \& Lafortune 2005) e entre as opções de vias de administração de anestésicos injetáveis estão as vias intravenosa, intramuscular e intraóssea, sendo a primeira de difícil acesso nessas espécies em decorrência de seu tamanho (Altman 1980, Harrison 1986). A administração intramuscular geralmente é realizada nos músculos peitorais, sendo que pequenos pássaros como pombos, periquitos e canários possuem pouca massa muscular peitoral (Vesal \& Zare 2006). Entre as desvantagens de aplicação intramuscular em aves estão a possibilidade de injeções inadvertidas na cavidade celomática ou por via intravascular, dor na aplicação e possibilidade de danos em terminações nervosas quando realizada em músculos da coxa (Moghadam et al. 2009).

A via de administração intranasal surge como uma alternativa em se evitar dor e ansiedade comparada à administração intramuscular, sendo utilizada em medicina pediátrica como uma via alternativa considerada segura e eficaz para administração de medicamentos (Wilton et al. 1988, Rey et al. 1991, Karl et al. 1992, Kendall 2001, Bhakta et al. 2007). Em medicina veterinária, a administração intranasal de anestésicos e suas combinações têm sido utilizadas em coelhos (Robertson 1994) e aves (Vesal \& Eskandari 2006, Vesal \& Zare 2006, Bigham \& Moghaddam 2009, Moghadam et al. 2009).

Os objetivos do presente estudo foram avaliar os efeitos anestésicos, bem como a viabilidade da associação de cetamina S+ e midazolam administrados pela via intranasal (IN) em comparação com a via intramuscular (IM) em pombas rolas.

\section{MATERIAL E MÉTODOS}

O presente estudo foi aprovado pelo Comitê de Ética e Bem-Estar Animal (CETEA) do Centro de Ciências Agroveterinárias, UDESC
(Protocolo 1.40/10). Foram utilizados 12 pombas do gênero Streptotelia sp. de ambos os sexos, adultas, pesando $136 \pm 9,7 \mathrm{~g}$, as quais foram mantidas em gaiolas (4 animais/gaiola) em ambiente com temperatura entre 18 e $20^{\circ} \mathrm{C}$ e com disponibilidade de ração comercial e água ad libitum. Antes de cada procedimento, jejum hídrico e sólido de duas horas foi realizado.

Como protocolo de anestesia dos animais, foram utilizados $3,5 \mathrm{mg} / \mathrm{kg}$ de midazolam (Dormire ${ }^{\circledR}$, Lab. Cristália, Itapira-SP) associados a $20 \mathrm{mg} / \mathrm{kg}$ de cetamina $\mathrm{S}+\left(\right.$ Ketamin $\mathrm{S}+{ }^{\circledR}$, lab. Cristália, Itapira-SP), os quais foram administrados de acordo com o grupo (estudo randomizado). Assim, os animais foram alocados em dois grupos de acordo com a via de administração empregada: grupo intramuscular (IM) ou grupo intranasal (IN). Cada animal foi utilizado como controle próprio, sendo respeitado um intervalo mínimo entre tratamentos de 15 dias.

Para administração dos fármacos por via intranasal, os animais foram contidos fisicamente em decúbito dorsal e posteriormente, aplicou-se lentamente os fármacos com o auxílio de uma micropipeta (P20-Eppendorf Research, São Paulo/SP). 0 midazolam foi depositado na narina direita e imediatamente após, a cetamina S+ foi aplicada na narina esquerda (Fig.1).

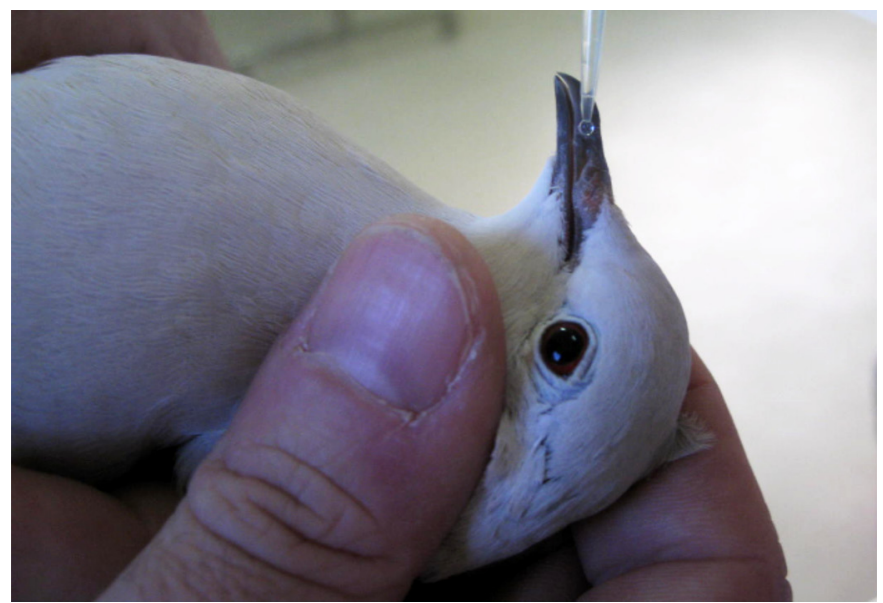

Fig.1. Administração intranasal de fármacos com utilização de micropipeta em pomba rola.

Após a administração dos fármacos, os animais foram colocados em decúbito dorsal e os seguintes parâmetros foram avaliados: (1) período de latência (avaliado em segundos e caracterizado como o tempo entre a aplicação dos fármacos e o surgimento dos primeiros sinais de anestesia, caracterizados por redução dos reflexos, fechamento dos olhos e possibilidade de manutenção da ave em decúbito dorsal); (2) duração de decúbito dorsal (avaliado em minutos, sendo o tempo compreendido desde o fim do período de latência até o retorno dos primeiros reflexos e a não permissão da permanência em decúbito dorsal); (3) tempo total da anestesia (avaliado em minutos, tempo compreendido desde o final do período de latência até a recuperação total, caracterizada pela presença de movimentos de cabeça, permanência no poleiro, movimentos voluntários e procura por comida); e (4) tempo de recuperação anestésica (tempo compreendido desde o fim do tempo de decúbito dorsal até o final do tempo total de anestesia). No período de recuperação os animais foram observados quanto a ocorrência de efeitos adversos como tremores musculares, movimentos exacerbados de asas ou pernas, presença ou ausência de regurgitação bem como era oferecido alimentação aos animais e observado a procura imediata ou não pelo alimento.

A avaliação de todos os parâmetros foi realizada sempre pelo mesmo avaliador, o qual desconhecia o tratamento utilizado. 
A análise estatística foi realizada por meio do teste estatístico de Wilcoxon. Todos os resultados estão expressos como mediana e valor de $1^{\circ}$ e $3^{\circ}$ quartil, sendo as diferenças consideradas significativas com valor de $\mathrm{P} \leq 0,05$.

\section{RESULTADOS}

Na comparação entre os grupos IM e IN, não foram observadas diferenças significativas para os parâmetros período de latência e duração de decúbito dorsal $(\mathrm{P} \leq 0,05)$. 0 período de latência obtido foi de 30 [30-47,5] e 40 [30-50] segundos para os grupos IM e IN, respectivamente, sendo que o tempo de duração de decúbito dorsal foi de 59 [53,25-65] e 63 [37-71,25] minutos para os grupos IM e IN, respectivamente. (Quadro 1).

\section{Quadro 1. Valores de mediana, 1 e 3울 quartil entre colchetes, referentes às variáveis período de latência (segundos), duração de decúbito dorsal (minutos), tempo de anestesia total (minutos) e tempo de recuperação (minutos) em pombos tratados com cetamina $S+(20 \mathrm{mg} / \mathrm{kg})$ e midazolam $(3,5 \mathrm{mg} / \mathrm{kg}$ ) pela via intranasal (IN) ou intramuscular (IM)

\begin{tabular}{lccc}
\hline \multirow{2}{*}{ Variáveis } & \multicolumn{3}{c}{ Grupos } \\
\cline { 2 - 4 } & IM & IN & Valor de P \\
\hline Período de latência (seg) & $30[30-47,5]$ & $40[30-50]$ & 0,938 \\
$\begin{array}{l}\text { Duração de decúbito dor- } \\
\text { sal (min) }\end{array}$ & $59[53,25-65]$ & $63[37-71,25]$ & 0,922 \\
$\begin{array}{l}\text { Tempo de anestesia total } \\
\text { (min) }\end{array}$ & $88[86,25-94,5]$ a & $68[53,5-93]$ b & 0,037 \\
$\begin{array}{l}\text { Tempo de recuperação } \\
\text { (min) }\end{array}$ & $32[28,25-38,25]$ a & $15[4,25-19,5]$ b & 0,014 \\
\hline
\end{tabular}

$\overline{a, b}$ Letras minúsculas diferentes entre colunas representam diferenças entre grupos pelo teste estatístico de Wilcoxon $(\mathrm{P} \leq 0,05)$.

0 tempo total de anestesia foi significativamente mais prolongado no grupo IM (88 [86,25-94,5] minutos), quando comparado ao grupo IN (68 [53,5-93] minutos) (Quadro 1).

Todos os animais aceitaram a administração intranasal sem resistência e após a recuperação total do animal foi observado a procura imediata por alimento na maioria dos animais de ambos os grupos.

A recuperação anestésica foi mais rápida no grupo IN (15 [4,25-19,5] minutos) comparada ao grupo IM (32 $[28,25-38,25]$ minutos). Não foram observados tremores musculares ou movimentos em nenhum animal dos grupos estudados, entretanto, dois animais de cada grupo apresentaram regurgitação na fase de recuperação.

\section{DISCUSSÃO}

A utilização intranasal da associação de cetamina S+ e midazolam para a anestesia de pombos mostrou-se eficaz, com curto período de latência e rápida recuperação em pombos.

Este estudo é o primeiro a avaliar a associação de cetamina S+ e midazolam pela via intranasal comparada com a via intramuscular para a anestesia de pombos e os resultados observados indicaram que a via intranasal é eficaz e pode ser útil em promover anestesia adequada para a realização de procedimentos terapêuticos de curta duração como curativos e medicações, sedação para procedimentos diagnósticos, troca de talas, entre outros.
As doses utilizadas neste ensaio foram escolhidas a partir de estudos pilotos de doses já descritas na literatura. A cetamina é recomendada na dose de 40 a $50 \mathrm{mg} / \mathrm{kg}$ e o midazolam, de 3,65 a 7,3mg/kg, dependendo das associações (Vesal \& Eskandari 2006). Nos estudos pilotos foram empregadas doses de 3,5 e $7,0 \mathrm{mg} / \mathrm{kg}$ de midazolam associados a 20 e $40 \mathrm{mg} / \mathrm{kg}$ de cetamina, entretanto, com as doses de $7,0 \mathrm{mg} / \mathrm{kg}$ de midazolam e $40 \mathrm{mg} / \mathrm{kg}$ de cetamina os animais apresentavam recuperação muito lenta com evidente depressão respiratória, assim, optou-se pelas doses menores.

A cetamina tem sido recomendada por vários autores como um agente viável em pássaros, porém, ela é raramente usada de forma isolada devido ao pobre relaxamento muscular, ocorrência de tremores musculares, contrações miotônicas e opistótono (Christensen et al. 1987, Mohammad et al. 1993). Esta substância é um derivado da fenciclidina que promove estado de anestesia dissociativa caracterizada por sedação, amnésia, analgesia e catalepsia, sendo esta propriedade a responsável pela sua associação com agonistas alfa-2 adrenérgicos, fenotiazínicos ou benzodiazepínicos para prevenção de delírios e produção de relaxamento muscular (Hall \& Clark 1991, Hartsfield 1992). Em medicina, é muito utilizada como medicação pré-anestésica pela via intranasal, com boa eficácia e sem efeitos adversos para sedação em crianças (Diaz 1997). A cetamina S+ é o enantiômero levógiro da cetamina racêmica e apresenta maior afinidade a receptores NMDA, maior potência analgésica e menos efeitos adversos com menores doses quando comparada a cetamina racêmica (Duque et al. 2008, Larenza et al. 2008a, 2008b). Pelas características peculiares da cetamina S+, optou-se, no presente estudo, pela utilização da menor dose deste fármaco $(20 \mathrm{mg} / \mathrm{kg})$ preconizada para anestesia de pombos.

Em estudos anteriores, a cetamina racêmica foi utilizada em doses superiores às de cetamina $\mathrm{S}+$ neste trabalho. Uzun et al. (2003) observaram depressão cardiorrespiratória acentuada e período de sedação que variou entre 15 e 60 minutos após $120 \mathrm{mg} / \mathrm{kg}$ de cetamina pela via intramuscular em pombos pré-medicados com medetomidina. Resultados semelhantes foram relatados por Atalan et al. (2002), com o uso de $100 \mathrm{mg} / \mathrm{kg}$ de cetamina após medicação pré-anestésica com butorfanol e medetomidina pela via intramuscular nesta mesma espécie. Mostachio et al. (2008) estudaram os efeitos da xilazina $(3 \mathrm{mg} / \mathrm{kg})$ seguida da associação de diazepam $(4 \mathrm{mg} / \mathrm{kg})$ e cetamina $(25 \mathrm{mg} / \mathrm{kg})$ em galos e constataram períodos de recuperação prolongados. Embora este estudo não tenha incluído a avaliação dos parâmetros fisiológicos, não foi observada depressão cardiorrespiratória evidente (apneia) como nos trabalhos citados anteriormente, o que pode ser explicado pelas menores doses de ambos os fármacos utilizadas, como também pelas diferentes associações de fármacos.

0 midazolam promove seus efeitos sedativos por agir em receptores $\mathrm{GABA}_{\mathrm{A}^{\prime}}$ o que potencializa os efeitos inibitórios do neurotransmissor GABA no sistema nervoso central (Skerritt \& Johnston 1983). Dentre as vantagens deste benzodiazepínico, o mesmo apresenta características hidrossolúveis, pode ser aplicado pela via intramuscular, 
promove mínimos efeitos cardiovasculares e possui maior potência e duração de ação mais curta em relação ao diazepam (Ludders \& Matthews 1996).

A utilização intramuscular de midazolam de forma isolada em pombos (Smith \& Muir 1992), gansos (Valverde et al. 1990), codornas (Colinus virginianus) (Day \& Roge 1996) e periquitos (Vesal \& Eskandari 2006) resultou em sedação de curta duração e estabilidade cardiovascular característica da classe destes fármacos, pela via intranasal, este benzodiazepínico tem seu uso na medicação pré-anestésica pediátrica, evitando o desconforto das vias intravenosa ou intramuscular (Louon \& Reddy 1994). Com a aplicação intranasal do midazolam em coelhos, observou-se redução na frequência respiratória, porém, sem afetar a saturação de oxihemoglobina (Robertson \& Eberhart 1994), este último achado também relatado em crianças (Karl et al 1992). Comparado ao diazepam, o midazolam apresenta mais rápido período de latência e duração de sedação em canários e mandarins sem depressão cardiorrespiratória evidente (Vesal \& Zare 2006, Bigham \& Moghaddam 2009). Outra vantagem do midazolam é que ele não causa mudanças no parênquima pulmonar, ao contrário do que foi observado com o uso de diazepam pela via endotraqueal em gatos resultando em mudanças histológicas deletérias (Rusli et al. 1987). Esta diferença de irritabilidade está associada ao propilenoglicol componente do veículo conservante do diazepam.

Efeitos adversos decorrentes da aplicação da associação midazolam e cetamina S+ não foram observadas em nenhum animal deste estudo, o que foi semelhante a outros estudos com benzodiazepínicos e da associação destes com cetamina pela via intranasal em outras aves (Vesal \& Eskandari 2006, Vesal \& Zare 2006). Apesar de realizado um período de jejum prévio, dois animais de cada grupo apresentaram regurgitação durante o período de recuperação, este pode ser considerado um efeito adverso de grande importância, pois a ocorrência durante o período de anestesia ou mesmo no período de recuperação anestésica pode provocar a ocorrência de pneumonia por falsa via considerando que as vias aéreas superiores ainda encontram-se relaxadas e que as aves são mais suscetíveis a refluxo devido á ausência de epiglote (Lierz \& Korbel 2012). Efeitos adversos, como sensação de queimação transitória e lacrimejamento, têm sido associados ao midazolam intranasal em humanos, o que está relacionado com o seu baixo $\mathrm{pH}(3,0-3,5)$ (Ng \& Sha 2006). A cetamina também possui $\mathrm{pH}$ baixo em torno de 3,5 o que pode levar a irritabilidade no local de administração e dor quando administrada pela via intramuscular (Valadão 2009). Entretanto tais efeitos como irritabilidade ou lacrimejamento não foram observados após a administração intranasal no presente estudo.

A mucosa intranasal é uma via com alta permeabilidade para a absorção de anestésicos, com rápida e completa absorção no homem (Robertson \& Eberhart 1994, Bjorkmann et al. 1997, Burstein et al. 1997, Kendall et al. 2001). O local exato de absorção da via intranasal não é totalmente elucidado, mas devido à grande vascularização desta região, os fármacos atingem rapidamente a circulação sistêmica. Este fator explica o curto período de latência observado no presente estudo, que foi semelhante à injeção intranasal de midazolam em canários (Vesal \& Zare 2006) e cetamina e midazolam em periquitos (Vesal \& Eskandari 2006). Alguns possíveis efeitos adversos da administração intranasal em aves incluem aerossaculite devido a contaminação dos sacos aéreos craniais devido a flora presente na cavidade nasal das aves

A biodisponibilidade média do midazolam após administração intranasal varia de $76 \pm 12 \%$, sendo a concentração sérica efetiva alcançada em menos de 10 minutos em crianças (Haschke et al. 2010). Outros estudos revelaram menor biodisponibilidade em humanos (55- 57\%), entretanto, sendo maior do que as vias oral ou retal, pois não há o metabolismo de primeira passagem (Wallbergh et al. 1991, Burstein et al. 1997).

Comparado com a administração intranasal de midazolam em coelhos, os quais apresentaram período de latência de três minutos e duração de sedação de 24 minutos (Robertson \& Eberhart 1994), este estudo revelou início de ação mais rápido e período mais longo de anestesia pela via intranasal. A utilização de midazolam em pombos pela via intranasal (Moghadam et al. 2009) apresentou início e duração de ação mais longos (3,2 e 82 minutos, respectivamente) comparados aos resultados deste estudo. Estas diferenças podem ser atribuídas pela associação de fármacos, onde a utilização de cetamina S+ no protocolo proposto no presente estudo promoveu um curto período de latência, assim como duração de ação mais curta, o que é esperado após administração intramuscular, porém observou-se ação semelhante pela via intranasal. Entretanto, em razão da redução das doses, a duração de ação foi menor comparada ao midazolam utilizado de forma isolada pela via intranasal em pombos (Moghadam et al. 2009). A combinação de cetamina e benzodiazepínicos melhora o relaxamento muscular, permite sedação adequada e melhora a qualidade da recuperação pela possibilidade do uso de doses reduzidas de ambos os fármacos (Varner et al. 2004). No período de recuperação em ambos os grupos observaram-se que os animais, após a recuperação total, estavam alertas e rapidamente buscavam por alimento, o que foi semelhante ao observado com o uso isolado de benzodiazepínicos por via intranasal em canários os quais no período de recuperação estavam famintos (Vesal \& Zare 2006). Esse achado de aumento de apetite, pode ser atribuído ao benzodiazepínico utilizado.

A associação intranasal de cetamina e midazolam foi utilizada em periquitos (Vesal \& Eskandari 2006) e resultou em início de ação de 2,2 minutos, duração de decúbito dorsal de 70,7 minutos e duração de sedação de 210,8 minutos. Estas observações foram contrastantes com os resultados aqui expostos, em que apesar da ocorrência de período de latência mais curto (40 segundos), resultou em duração de decúbito dorsal e de anestesia mais rápidos (63 e 68 minutos, respectivamente). Esta discrepância entre resultados pode ser decorrente da grande variedade intra e interespécies de pássaros (Samour et al. 1984), considerando que diferentes famílias de pássaros foram avaliadas nos outros estudos, o que pode resultar em particularidades na biotransformação dos fármacos e na ação farmaco- 
lógica destes. Outra diferença importante é que a dose da cetamina S+ deste estudo $(20 \mathrm{mg} / \mathrm{kg})$ foi metade da dose utilizada em periquitos ( $40 \mathrm{mg} / \mathrm{kg}$ ), o que também poderia justificar a menor duração de ação nos pombos, apesar de alguns autores afirmarem que a cetamina S+, por apresentar maior efeito analgésico e maior potência, pode ter sua dose reduzida como o observado para gatos, cães e pôneis (Duque et al. 2008, Larenza et al. 2008a, 2008b).

A associação de cetamina S+ e midazolam pela via intranasal apresentou tempo total de anestesia menor quando comparada à via intramuscular. Esse efeito resultou em recuperação anestésica mais rápida, o que dependendo do procedimento pode ser muito vantajoso. As principais desvantagens de sedações ou anestesias prolongadas em aves é que durante o procedimento ocorre restrição alimentar e perda de calor, os quais predispõem à hipotermia e hipoglicemia, considerando que essa classe possui pobre reserva de glicogênio hepático e alta taxa metabólica (Harrison 1986, Atalan et al. 2002).

\section{CONCLUSÃO}

A administração intranasal da associação de cetamina S+e midazolam mostrou-se como um método viável, aceitável, bem tolerado, e que produziu anestesia rápida e eficaz, de maneira similar à via intramuscular em pombas do gênero Streptotelia sp.

\section{REFERÊNCIAS}

Altman R. B. 1980. Avian anaesthesia. Compend. Contin. Educ. Pract. Vet. 2:38-42.

Atalan G., Uzun M., Demirkan I., Yildiz S. \& Cenesiz M. 2002. Effect of medetomidine-butorphanol-ketamine anaesthesia and atipamezole on heart and respiratory rate and cloacal temperature of domestic pigeons. J. Vet. Med. A 49:281-285.

Bhakta P., Ghosh B.R., Roy M. \& Mukherjee G. 2007. Evaluation for intranasal midazolam for preanesthetic sedation in pediatric patients. Indian J. Anaesth. 51(2):111-116.

Bigham A.S. \& Moghaddam A.K.Z. 2009. Finch (Taeneopygia guttata) sedation with intranasal administration of diazepam, midazolam or xylazine. J. Vet. Pharmacol. Therap. 1-3 doi: 10.1111/j.1365-2885.2009.01102.x

Bojrkman S., Rigemar G. \& Idvall J. 1997. Pharmacokinetics of midazolam given as intranasal spray to adult surgical patients. Brit. J. Anaesth. 79: 575-80.

Burstein A.H., Modica R., Hatton M., Forrest A. \& Gengo F.M. 1997. Pharmacokinetics and pharmacodynamics of midazolam after intranasal administration. J. Clin. Pharmacol. 37:711-718.

Coles B.H. 1997. Anaesthesia, p.125-147. In: Altman R.B., Clubb S.L., Dorrestein G.M. \& Quesenberry K. (Eds), Avian Medicine and Surgery. $2^{\text {nd }}$ ed. Blackwell Science, Oxford.

Christensen J., Fosse R.T., Halvorsen O.J. \& Morild I. 1987. Comparison of various anesthetic regimens in the domestic fowl. Am. J. Vet. Res. 48:1649-1657.

Day T.K. \& Roge C.K. 1996. Evaluation of sedation in quail induced by use of midazolam and reversed by use of flumazenil. J. Am. Vet. Med. Assoc. 209:969-971.

Diaz J.H. 1997. Intranasal ketamine preinduction of paediatric outpatients. Pediatr. Anaesth. 7(4):273-278.

Duque J.C., Oleskovicz N., Guirro E.C.B.P., Valadão C.A.A. \& Soares V.E. 2008. Relative potency of ketamine and S(+)-ketamine in dogs. J. Vet. Pharmacol. Therap. 31:344-348.

Gleed R.D. \& Ludders J.W. 2001. Recent Advances in Veterinary Anesthesia and Analgesia: companion animals. International Veterinary Information Service, Ithaca, NY.

Gunkel C. \& Lafortune M. 2005. Current techniques in avian anesthesia. Seminars in Avian and Exotic Pet Medicine 4:263-276.

Hartsfield S.M. 1992. Advantages and guidelines for using ketamine for induction of anaesthesia. Vet. Clin. North Am., Small Anim. Pract. 22:266267.

Harrison G.J. 1986. Anesthesiology, p.549-559. In: Harrison G.J. \& Harrison L.R. (Eds), Clinical Avian Medicine and Surgery. W.B. Saunders, Philadelphia.

Hall L.W. \& Clarke K.W. 1991. Anaesthesia in the dog, p.51-79. In: Hall L.W. \& Clarke K.W (Eds), Veterinary Anaesthesia,. Bailliere Tindall, Philadelphia.

Kendall J.M., Reeves B.C. \& Latter V.S. 2001. Multicenter randomized controlled trial of nasal diamorphine for analgesia in children and teenagers with clinical fractures. Brit. Med. J. 322:261-265.

Karl H.W., Keifer A.T., Rosenberger J.L., Laranch M.G. \& Ruffle J.M. 1992. Comparison of the safety and efficacy of intranasal midazolam or sufentanil for preinduction of anesthesia in pediatric patients. Anesthesiology 76:209-215.

Kendall J.M., Reeves B.C. \& Latter V.S. 2001. Multicenter randomized controlled trial of nasal diamorphine for analgesia in children and teenagers with clinical fractures. Brit. Med. J. 322:261-265.

Larenza M.P., Althaus H., Conrot A., Balmer C., Schatzmann U. \& Bettschart-Wolfensberger R. 2008a. Anaesthesia recovery quality after racemic ketamine or S-ketamine administration to male cats undergoing neutering surgery. Schweiz. Arch. Tierheilkd. 150:599-607.

Larenza M.P., Knobloch M., Landoni M.F., Levionnois O.L., Kronen P.W., Theurillat R., Schatzmann U. \& Thormann W. 2008b. Stereoselective pharmacokinetics of ketamine and norketamine after racemic ketamine or S-ketamine administration in Shetland ponies sedated with xylazine. Vet. J. 177:432-435.

Lauretti G.R., Lima I.C.P.R., Buscatti R.Y. \& Reis M.P. 2000. Avaliação clínica dos efeitos hemodinâmicos, analgésicos, psicodélicos e de bloqueio neuromuscular da cetamina racêmica e de seu S(+) isômero. Revta Bras. Anestesiol. 50:357-362.

Lierz M. \& Korbel R. 2012. Anesthesia and analgesia in birds. J. Exotic Pet Med. 21:44-58.

Louon A. \& Reddy V.G. 1994. Nasal midazolam and ketamine for pediatric sedation during computerized tomography. Acta Anaesthesiol. Scand. 38:259-261.

Ludders J.W. \& Matthews N. 1996. Anesthesia and immobilization of birds, p.645-669. In: Thurmon J.C., Tranquilli W.J. \& Benson G.J. (Eds), Lumb and Jones' Veterinary Anesthesia. $3^{\text {rd }}$ ed. William and Wilkins, Baltimore, MD.

Haschke M., Suter K., Hofmann S., Witschi R., Fröhlich J., Imanidis G., Drewe J., Briellmann T.A., Dussy F.E., Krärenbühl S. \& Surber D. 2010. Pharmacokinetics and pharmacodynamics of nasally delivered midazolam. Brit. J. Clin. Pharmacol. 69:607-616.

Moghadam A.Z., Sadegh A.B., Sharifi S. \& Habibian S. 2009. Comparison of intranasal administration of diazepam, midazolam and xylazine in Pigeons: clinical evaluation. Iranian J. Vet. Sci. Tech. 1:19-26.

Mohammad F.K., Al-Badrany M.S. \& Al-Hasan A.M. 1993. Detomidine-ketamine anaesthesia in chickens. Vet. Rec. 133:192.

Mostachio G.Q., De-Oliveira L.D., Carciofi A.C. \& Vicente W.R.R. 2008. The effects of anesthesia with a combination of intramuscular xylazine-diazepam-ketamine on heart rate, respiratory rate and cloacal temperature in roosters. Vet. Anaesth. Analg. 35:232-236.

Ng E. \& Shah V. 2006. Benzodiazepines for Sedation in Infants and Children, p.170-183. In: Lader H., Cardinali D.P. \& Pandi-Perumal S.R. (Eds), Sleep and Sleep Disorders: a neuropsychopharmacological approach. Springer, London.

Pfenninger E.G., Durieux M.E. \& Himmelseher S. 2002. Cognitive impairment after small-close ketamine isomers in comparison to equianalgesic racemic ketamine in human volunteers. Anesthesiology 96:357-366. 
Rey E., Delaunay L., Pons G., Murat I., Richard M.O., Saint-Maurice C. \& Olive G. 1991. Pharmacokinetics of midazolam in children: Comparative study of intranasal and intravenous administration. Eur. J. Clin. Pharmacol. 41:355-357.

Robertson S.A. \& Eberhart S. 1994. Efficacy of the intranasal route for administration of anesthetic agents to adult rabbits. Lab. Anim. Sci. 38:259-261.

Rusli M., Spivey W.H., Bonner H., MacNamara R.M., Aaron C.K. \& Lathers C.M. 1987. Endotracheal diazepam: absorption and pulmonary pathologic effects. Ann. Emerg. Med. 16:314-318.

Samour J.H., Jones D.M. \& Knight J.A. 1984. Comparative studies of the use of some injectable anesthetic agents in birds. Vet. Rec. 115:6-11.

Skerritt J.H. \& Johnston G.A. 1983. Enhancement of GABA binding by benzodiazepines and related anxiolytics. Eur. J. Pharmacol. 89:193-198.

Smith J. \& Muir W.W. 1992 Cardiopulmonary effects of midazolam and flumazenil in racing pigeons. Vet. Surg. 21:499.

Uzun M., Yildiz S., Atalan G., Kaya M. \& Sulu N. 2003. Effects of medetomidine-ketamine combination anaesthesia on electrocardiographic findings, body temperature, and heart and respiratory rates in domestic pigeons. Turkey J. Vet. Anim. Sci. 27:377-382.

Valadão C.A.A. 2009. Anestésicos dissociativos, p.237-245. In: Fantoni D.T. \& Cortopassi S.R.G. (Eds), Anestesia em Cães e Gatos. $2^{\underline{a}}$ ed. Roca, São Paulo,
Valverde A., Honeyman V.L., Smith D.A., Dyson D.H. \& Valliant A.E. 1990. Determination of a sedative dose and influence of midazolam on cardiopulmonary function in Canada geese. Am. J. Vet. Res. 51:1071-1074.

Varner J., Clifton K.R., Poulos S., Broderson J.R. \& Wyatt R.D. 2004. Lack of efficacy of injectable ketamine with xylazine or diazepam for anesthesia in chickens. Lab. Anim. 33:36-39.

Vesal N. \& Zare P. 2006. Clinical evaluation of intranasal benzodiazepines, $\alpha 2$-agonists and their antagonists in canaries. Vet. Anaesth. Analg. 33: 143-148.

Vesal N. \& Eskandari M.H. 2006. Sedatives effects of midazolam and xylazine with or without ketamine and detomidine alone following intranasal administration in Ring-necked parakeets. J. Am. Vet. Med. Assoc. 228:383-388.

Walbergh E.J., Wills R.J. \& Eckhert J. 1991. Plasma concentrations of midazolam in children following intranasal administration. Anesthesiology 74:233-235.

Wilton N.C.T., Leigh J., Rosen D.R. \& Pandit U.A. 1988. Preanesthetic sedation of preschool children using intranasal midazolam. Anesthesiology 69:972-975

White P.F., Ham J., Way W.L. \& Trevor A. 1980. Pharmacology of ketamine isomers in surgical patients. Anesthesiology 52:231-239. 